\title{
Uma análise de pressupostos teóricos da abordagem C-T-S (Ciência - Tecnologia - Sociedade) no contexto da educação brasileira
}

\author{
Wildson Luiz Pereira dos Santos ${ }^{1}$ \\ Eduardo Fleury Mortimer²
}

\section{Resumo:}

Desde a década de sessenta, currículos de ensino de ciências com ênfase em CTS - ciência, tecnologia e sociedade - vêm sendo desenvolvidos no mundo inteiro. Tais currículos apresentam como objetivo central preparar os alunos para o exercício da cidadania e caracterizam-se por uma abordagem dos conteúdos científicos no seu contexto social. No presente artigo, discutimos criticamente os pressupostos desses currículos, de modo a fornecer subsídios para a elaboração de novos modelos curriculares na área de Ciências da Natureza e suas Tecnologias os quais possam contemplar a finalidade da educação básica em preparar o aluno para o exercício consciente da cidadania.

\begin{abstract}
:
Since the 60s, science curricula with emphasis in STS - Science, Technology and Society have been developed all around the world. These curricula aim, mainly, at preparing students to the exercise of the citizenship and are characterized by approaching science content in the social context. In this article we discuss critically the presupposition of such curricula as to provide support for the elaboration of new curricular models in the area of Natural Science and its Technologies which can contribute to the aim of Primary and Secondary Education of preparing the students to the conscious exercise of citizenship.
\end{abstract}

\footnotetext{
1 Professor da Universidade de Brasília / UNB, Instituto de Química

2 Professor da Universidade Federal de Minas Gerais, Faculdade de Educação, Cecimig
} 


\section{Introdução}

Vivemos hoje em um mundo notadamente influenciado pela ciência e tecnologia. Tal influência é tão grande que podemos falar em uma autonomização da razão científica em todas as esferas do comportamento humano. Essa autonomização resultou em uma verdadeira fé no homem, na ciência, na razão, enfim, uma fé no progresso (BERNARD e CROMMELINCK, 1992). As sociedades modernas passaram a confiar na ciência e na tecnologia como se confia em uma divindade. A lógica do comportamento humano passou a ser a lógica da eficácia tecnológica e suas razões passaram a ser as da ciência (BAZZO, 1998).

Como conseqüência do cientificismo que emerge desse processo, a supervalorização da ciência gerou o mito da salvação da humanidade, ao considerar que todos os problemas humanos podem ser resolvidos cientificamente. Uma outra conseqüência é o mito da neutralidade científica (JAPIASSU, 1999). Tais crenças tiveram repercussões no ensino de ciências, por exemplo, a orientação curricular de formar um minicientista por meio da vivência do "método científico", que teve grande influência sobre o ensino de ciências a partir do final dos anos 50 .

O cientificismo tem também uma função ideológica de dominação. Segundo HABERMAS (1983), com o desenvolvimento do modo de produção capitalista, houve uma cientificização da técnica e, nesse processo, o desenvolvimento tecnológico passou a depender de um sistema institucional no qual conhecimento técnico e científico são interdependentes. Ainda, segundo esse autor, "com a investigação industrial de grande escala, ciência, técnica e valorização foram inseridas no mesmo sistema" (: 330). HABERMAS defende, assim, a tese central de Marcuse de que a ciência e a técnica cumprem a função de legitimação da dominação, pois as metodologias científicas levam a uma dominação da natureza com uma eficácia cada vez maior, proporcionando os instrumentos para uma dominação cada vez mais eficiente do homem sobre o homem.

Ao invés de as necessidades humanas definirem as necessidades de produção - o que seria a norma para uma sociedade verdadeiramente humana - são as necessidades do funcionamento do sistema que irão criar as "falsas necessidades" de consumo (..) E o sistema criou o homem à sua imagem e semelhança e lhe disse: Não terás outros deuses diante de mim! (ALVES, 1968: 20).

Estudos da filosofia e da sociologia da ciência vêm demonstrando a falácia do mito cientificista. Não existe a neutralidade científica nem a ciência é eficaz para resolver as grandes questões éticas e sócio-políticas da humanidade (FOUREZ, 1995; JAPIASSU, 1999). Além disso, a ciência e a tecnologia têm interferido no ambiente e suas aplicações têm sido objeto de muitos debates éticos, o que torna inconcebível a idéia de uma ciência pela ciência, sem consideração de seus efeitos e aplicações. É nesse contexto que estudos sobre ciência, tecnologia e sociedade têm recebido uma grande atenção, sobretudo no período posterior ao da Segunda Guerra Mundial e, nas últimas décadas, vêm influenciando a elaboração de currículos de ciências no mundo inteiro (BRIDGSTOCK et al., 1998).

É nesse contexto também que emergiu um novo modo de produção do conhecimento 
(GIBBONS et al, 1994), o qual tem-se desenvolvido em um contexto de aplicação, com características mais transdisciplinares do que disciplinares e dando lugar a uma interação entre diferentes atores sociais, como cientistas, representantes dos governos, do setor produtivo, de organizações não-governamentais e da imprensa. Esse novo modo de produção tem acarretado um aumento da responsabilidade social dos produtores de conhecimento científico e tecnológico. Nele os diferentes profissionais se unem no interesse comum de resolver grandes problemas, como a cura da AIDS, a escassez ou má distribuição de alimentos, etc. Isso passa a exigir do novo cientista uma maior reflexão e, sobretudo, a capacidade de dialogar com outras áreas para participar da análise de tais problemas em uma perspectiva multidisciplinar.

Há de se considerar, ainda, que as decisões sobre as aplicações da ciência deveriam passar por um filtro social (VARGAS, 1994). Para VARGAS

uma nação adquire autonomia tecnológica não necessariamente quando domina um ramo de alta tecnologia; mas quando consegue uma ampla e harmoniosa interação entre esses subsistemas tecnológicos, sob o controle, orientação e decisão dos 'filtros sociais"(:186).

Alfabetizar, portanto, os cidadãos em ciência e tecnologia é hoje uma necessidade do mundo contemporâneo (SANTOS e SCHNETZLER, 1997). Não se trata de mostrar as maravilhas da ciência, como a mídia já o faz, mas de disponibilizar as representações que permitam ao cidadão agir, tomar decisão e compreender o que está em jogo no discurso dos especialistas (FOUREZ, 1995). Essa tem sido a principal proposição dos currículos com ênfase em CTS.

\section{O que é currículo com ênfase em CTS?}

ROBERTS (1991) refere-se às ênfases curriculares "Ciência no contexto social" e "CTS" como aquelas que tratam das inter-relações entre explicação científica, planejamento tecnológico e solução de problemas, e tomada de decisão sobre temas práticos de importância social. Tais currículos apresentam uma concepção de: (i) ciência como atividade humana que tenta controlar o ambiente e a nós mesmos, e que é intimamente relacionada à tecnologia e às questões sociais; (ii) sociedade que busca desenvolver, no público em geral e também nos cientistas, uma visão operacional sofisticada de como são tomadas decisões sobre problemas sociais relacionados à ciência e tecnologia; (iii) aluno como alguém que seja preparado para tomar decisões inteligentes e que compreenda a base científica da tecnologia e a base prática das decisões; e (iv) professor como aquele que desenvolve o conhecimento de e o comprometimento com as inter-relações complexas entre ciência, tecnologia e decisões.

BYBEE (1987) caracteriza a orientação curricular de CTS como pesquisa e desenvolvimento de currículos que contemplem, entre outros: (i) a apresentação de conhecimentos e habilidades científicos e tecnológicos em um contexto pessoal e social; (ii) a inclusão de conhecimentos e habilidades tecnológicos; (iii) a ampliação dos processos de 
investigação de modo a incluir a tomada de decisão e (iv) a implementação de projetos de CTS no sistema escolar.

Segundo HOFSTEIN, AIKENHEAD e RIQUARTS (1988: 358), CTS pode ser caracterizado como o ensino do conteúdo de ciências no contexto autêntico do seu meio tecnológico e social, no qual os estudantes integram o conhecimento científico com a tecnologia e o mundo social de suas experiências do dia-a-dia. A proposta curricular de CTS corresponderia, portanto, a uma integração entre educação científica, tecnológica e social, em que os conteúdos científicos e tecnológicos são estudados juntamente com a discussão de seus aspectos históricos, éticos, políticos e sócio-econômicos (LÓPEZ e CEREZO, 1996).

\section{Breve histórico}

O agravamento dos problemas ambientais pós-guerra, a tomada de consciência de muitos intelectuais com relação às questões éticas, a qualidade de vida da sociedade industrializada, a necessidade da participação popular nas decisões públicas, estas cada vez mais sob o controle de uma elite que detém o conhecimento científico e, sobretudo, o medo e a frustração decorrentes dos excessos tecnológicos propiciaram as condições para o surgimento de propostas de ensino CTS (WAKS, 1990). Estudos na área da epistemologia da ciência, que incorporaram questões relativas aos aspectos econômicos e políticos da ciência, também contribuíram para o aparecimento dessa ênfase.

Os trabalhos curriculares em CTS surgiram, assim, como decorrência da necessidade de formar o cidadão em ciência e tecnologia, o que não vinha sendo alcançado adequadamente pelo ensino convencional de ciências. O cenário em que tais currículos foram desenvolvidos corresponde, no entanto, ao dos países industrializados, na Europa, nos Estados Unidos, no Canadá e na Austrália, em que havia necessidades prementes quanto à educação científica e tecnológica (LAYTON, 1994).

KRASILCHIK (1987), ao discutir a evolução da inovação educacional dos currículos de ciências no Brasil no período de 1950 a 1985, assinala que, na década de setenta, os mesmos começaram a incorporar uma visão de ciência como produto do contexto econômico, político e social. Já na década de oitenta, a renovação do ensino de ciências passou a se orientar pelo objetivo de analisar as implicações sociais do desenvolvimento científico e tecnológico.

Vários materiais didáticos e projetos curriculares brasileiros foram elaborados, incorporando elementos dessa perspectiva. Dentre os materiais didáticos, podemos citar: o projeto Unidades Modulares de Química (AMBROGl et al., 1987), as propostas pedagógicas de LUTFI (1988 e 1992), a coleção de livros do Grupo de Pesquisa em Ensino de Química da USP - GEPEQ, (1993, 1995, 1998), a coleção de livros de física do GREF (1990, 1991 e 1993), o livro Química na Sociedade (MÓL e SANTOS, 2000) e o livro Química, Energia e Ambiente (MORTIMER, MACHADO e ROMANELLI, 1999). Dentre as recomendações curriculares, podem ser destacadas a Proposta Curricular de Ensino de Química da CENP/SE do Estado de São Paulo (SÃO PAULO, 1988), as recomendações para o currículo do magistério de CISCATO e BELTRAN (1991), e a Proposta Curricular de Química para o Ensino Médio do Estado de Minas (MORTIMER, MACHADO e ROMANELLI, 1998).

Ainda sobre trabalhos de CTS no Brasil, pode-se citar a realização, em 1990, da 
"Conferência Internacional Ensino de Ciências para o Século XXI: ACT - Alfabetização em Ciência e Tecnologia", cuja temática central foi a educação científica dos cidadãos. Pode-se considerar, também, que a atual reforma curricular do ensino médio incorpora, em seus objetivos e fundamentos, elementos dos currículos com ênfase em CTS.

\section{Elementos curriculares}

\section{a) Objetivos}

O objetivo central da educação de CTS no ensino médio é desenvolver a alfabetização científica e tecnológica dos cidadãos, auxiliando o aluno a construir conhecimentos, habilidades e valores necessários para tomar decisões responsáveis sobre questões de ciência e tecnologia na sociedade e atuar na solução de tais questões (AIKENHEAD, 1994a; IGLESIA, 1995; HOLMAN, 1988; RUBBA e WIESENMAYER, 1988; SOLOMON, 1993b; YAGER, 1990; ZOLLER, 1982). As propostas identificam, assim, três objetivos gerais: (1) aquisição de conhecimentos, (2) utilização de habilidades e (3) desenvolvimento de valores (BYBEE, 1987).

Dentre os conhecimentos e as habilidades a serem desenvolvidos, HOFSTEIN, AIKENHEAD e RIQUARTS (1988) incluem: a auto-estima, a comunicação escrita e oral, o pensamento lógico e racional para solucionar problemas, a tomada de decisão, o aprendizado colaborativo/cooperativo, a responsabilidade social, o exercício da cidadania, a flexibilidade cognitiva e o interesse em atuar em questões sociais.

Nessa perspectiva, McCONNELL (1982) aponta que

Tomada de decisão pública pelos cidadãos em uma democracia requer: uma atitude cuidadosa, habilidades de obtenção e uso de conhecimentos relevantes, consciência e compromisso com valores e capacidade de transformar atitudes, habilidades e valores em ação. Todos esses passos podem ser encorajados se uma perspectiva de tomada de decisão for incorporada ao processo educacional (: 13) [tradução nossa].

Destaca-se, portanto, entre os objetivos, o desenvolvimento de valores. Esses valores estão vinculados aos interesses coletivos, como os de solidariedade, de fraternidade, de consciência do compromisso social, de reciprocidade, de respeito ao próximo e de generosidade. Tais valores são, assim, relacionados às necessidades humanas, o que significa um questionamento à ordem capitalista, na qual os valores econômicos se impõem aos demais.

Será por meio da discussão desses valores que contribuiremos na formação de cidadãos críticos comprometidos com a sociedade. As pessoas, por exemplo, lidam diariamente com dezenas de produtos químicos e têm que decidir qual devem consumir e como fazê-lo. Essa decisão poderia ser tomada levando-se em conta não só a eficiência dos produtos para os fins que se desejam, mas também os seus efeitos sobre a saúde, os seus efeitos ambientais, o seu valor econômico, as questões éticas relacionadas a sua produção e comercialização. Por exemplo, poderia ser considerado pelo cidadão, na hora de consumir determinado produto, se, na sua produção, é usada mão-de-obra infantil ou se os trabalhadores são explorados de maneira desumana; se, em alguma fase, da produção ao descarte, o produto agride o ambiente; se ele é objeto de contrabando ou de outra contravenção, etc. Certamente o cidadão 
não tem acesso a todas essas informações, mas refletir sobre tais questões significa mudar a postura em relação ao consumo de mercadorias, pois, em geral, na maioria das vezes, a decisão entre consumir um ou outro produto é tomada em função de sua aparência e qualidade, e quase nunca são considerados os aspectos sociais, ambientais e éticos envolvidos na sua produção. Considerações de tal ordem poderiam, por exemplo, resultar na diminuição, a longo prazo, do consumo de embalagens descartáveis, de produtos que agridem a camada de ozônio, etc., forçando uma reformulação drástica nos processos de fabricação.

\section{b) Estrutura conceitual}

A estrutura conceitual dos curso de CTS, resumida por BYBEE (1987), é composta pelos seguintes temas: conceitos científicos e tecnológicos, processos de investigação e interações entre ciência, tecnologia e sociedade. A aquisição de conhecimentos científicos e tecnológicos enfatizaria aspectos relacionados ao interesse pessoal, à preocupação cívica e às perspectivas culturais. Os processos de investigação científica e tecnológica propiciariam a participação ativa dos alunos na obtenção de informações, solução de problemas e tomada de decisão. A interação entre ciência, tecnologia e sociedade propiciaria o desenvolvimento de valores e idéias por meio de estudos de temas locais, políticas públicas e temas globais. Nesse sentido, consideramos relevante discutir que visões os currículos CTS apresentam sobre ciência, tecnologia, sociedade e suas inter-relações.

\section{Ciência}

Uma visão crítica da ciência, expressa tanto por filósofos quanto por sociólogos, tem buscado desfazer o mito do cientificismo que ideologicamente ajudou a consolidar a submissão da ciência aos interesses de mercado, à busca do lucro.

CHALMERS (1994), analisando trabalhos de diferentes epistemólogos e filósofos da ciência, discute os limites da ciência e o significado das suas dimensões sociais e políticas, fazendo uma crítica à pseudociência, ao método experimental e à objetividade científica, ao considerar a ciência, não como um corpus rígido e fechado, mas como uma atividade aberta que está em contínua construção.

LATOUR e WOOLGAR (1997), como representantes da corrente construtivista em sociologia, procuram demonstrar, em seus trabalhos em micro-etnografia das práticas científicas cotidianas, como os fatos da ciência, apesar de sua aparência objetiva e neutra, são, na verdade, construídos socialmente. Esses autores defendem a tese de que a ciência não é justificada somente por critérios racionais e cognitivos, pois esses critérios são também construídos socialmente pelos diferentes atores que participam da investigação científica. Com essa tese, eles questionam a visão mítica da ciência e de seus métodos, a sua a-historicidade, a sua universalidade, a natureza absoluta de suas técnicas e de seus resultados.

Além disso, a partir dessa posição relativista, LATOUR e WOOLGAR afirmam que não existe uma forma objetiva de avaliar se o conhecimento científico é um reflexo "verdadeiro" do mundo, e que a noção de "progresso" científico é, portanto, problemática. Esse aparente "irracionalismo" e relativismo da ciência é, no momento, uma questão polêmica e em aberto na sociologia e filosofia das ciências. Admitir que o conhecimento científico é socialmente construído não implica necessariamente adotar uma posição relativista. Parece-nos que o 
conhecimento científico é limitado também pela própria estrutura do mundo real, e que o progresso científico tem base empírica, embora seja socialmente construído e validado (DRIVER, ASOKO, LEACH, MORTIMER e SCOTT, 1994).

Nesse sentido, diversas pesquisas têm constatado que a compreensão da natureza da ciência é fundamental para que o aluno possa entender as suas implicações sociais (AIKENHEAD, 1985 e 1994a; LAYTON, DAVEY e JENKINS, 1986; RAMSEY, 1993; SOLOMON, 1993b; STIEFEL, 1995). Isso remete à necessidade de que, no currículo, sejam discutidos aspectos relacionados à filosofia, história e sociologia das ciências.

Dessa forma, SOLOMON (1988) propõe que os cursos de CTS deveriam apontar para o caráter provisório e incerto das teorias científicas. Com tal compreensão, os alunos poderiam avaliar as aplicações da ciência, levando em conta as opiniões controvertidas dos especialistas. Ao contrário, com uma visão de ciência como algo absolutamente verdadeiro e acabado, os alunos terão dificuldade de aceitar a possibilidade de duas ou mais alternativas para resolver um determinado problema.

O conteúdo referente às ciências dos currículos de CTS incluem, assim, aspectos relativos a estudos políticos de ciência, mais vinculados às questões sociais externas à comunidade científica (conservação de energia, crescimento populacional, efeitos da energia nuclear, etc.) e a aspectos da ciência vinculados às questões internas à comunidade científica, relacionadas a sua epistemologia e filosofia (ROSENTHAL, 1989).

Nessa perspectiva, ROSENTHAL (1989) apresenta uma série de aspectos relativos a ciências que poderiam ser abordados nos currículos, como questões de natureza:

1. filosófica - que incluiria, entre outros, aspectos éticos do trabalho científico, o impacto das descobertas científicas sobre a sociedade e a responsabilidade social dos cientistas no exercício de suas atividades;

2. sociológica - que incluiria a discussão sobre as influências da ciência e tecnologia sobre a sociedade e dessa última sobre o progresso científico e tecnológico; e as limitações e possibilidades de se usar a ciência e a tecnologia para resolver problemas sociais;

3. histórica - que incluiria discutir a influência da atividade científica e tecnológica na história da humanidade, bem como os efeitos de eventos históricos no crescimento da ciência e da tecnologia;

4. política - que passa pelas interações entre a ciência e a tecnologia e os sistemas público, de governo e legal; a tomada de decisão sobre ciência e tecnologia; o uso político da ciência e tecnologia; ciência, tecnologia, defesa nacional e políticas globais;

5. econômica - com foco nas interações entre condições econômicas e a ciência e a tecnologia, contribuições dessas atividades para o desenvolvimento econômico e industrial, tecnologia e indústria, consumismo, emprego em ciência e tecnologia, e

6. humanística - aspectos estéticos, criativos e culturais da atividade científica, os efeitos do desenvolvimento científico sobre a literatura e as artes, e a influência da humanidades na ciência e tecnologia.

Sendo assim, os conteúdos dos currículos CTS apresentam uma abordagem de ciência em sua dimensão ampla, em que são discutidos muitos outros aspectos além da natureza da 
investigação científica e do significado dos conceitos científicos. Tal abordagem de ciências contribui também para aqueles que pretendem ingressar na carreira científica, que vão se deparar com um novo modo de produção (GIBBONS,1994) que exige uma visão cada vez mais multidisciplinar e reflexiva das ciências.

Isso diferencia-se do modismo do assim chamado ensino do cotidiano, que se limita a nomear cientificamente as diferentes espécies de animais e vegetais, os produtos químicos de uso diário e os processos físicos envolvidos no funcionamento dos aparelhos eletro-eletrônicos. Um ensino que contemple apenas aspectos dessa natureza seria, a nosso ver, puramente enciclopédico, favorecendo uma cultura de almanaque. Essa seria uma forma de "dourar a pílula", ou seja, de introduzir alguma aplicação apenas para disfarçar a abstração excessiva de um ensino puramente conceitual, deixando, à margem, os reais problemas sociais.

\section{Tecnologia}

A tecnologia pode ser compreendida como o conhecimento que nos permite controlar e modificar o mundo. Atualmente a tecnologia está associada diretamente ao conhecimento científico, de forma que hoje tecnologia e ciência são termos indissociáveis. Isso tem levado a uma confusão comum que é reduzir a tecnologia à dimensão de ciência aplicada.

A tecnologia consiste em um conjunto de atividades humanas, associadas a sistemas de símbolos, instrumentos e máquinas, visando à construção de obras e à fabricação de produtos por meio de conhecimento sistematizado (VARGAS, 1994). O diagrama da FIG. 1 caracteriza essa concepção de tecnologia.

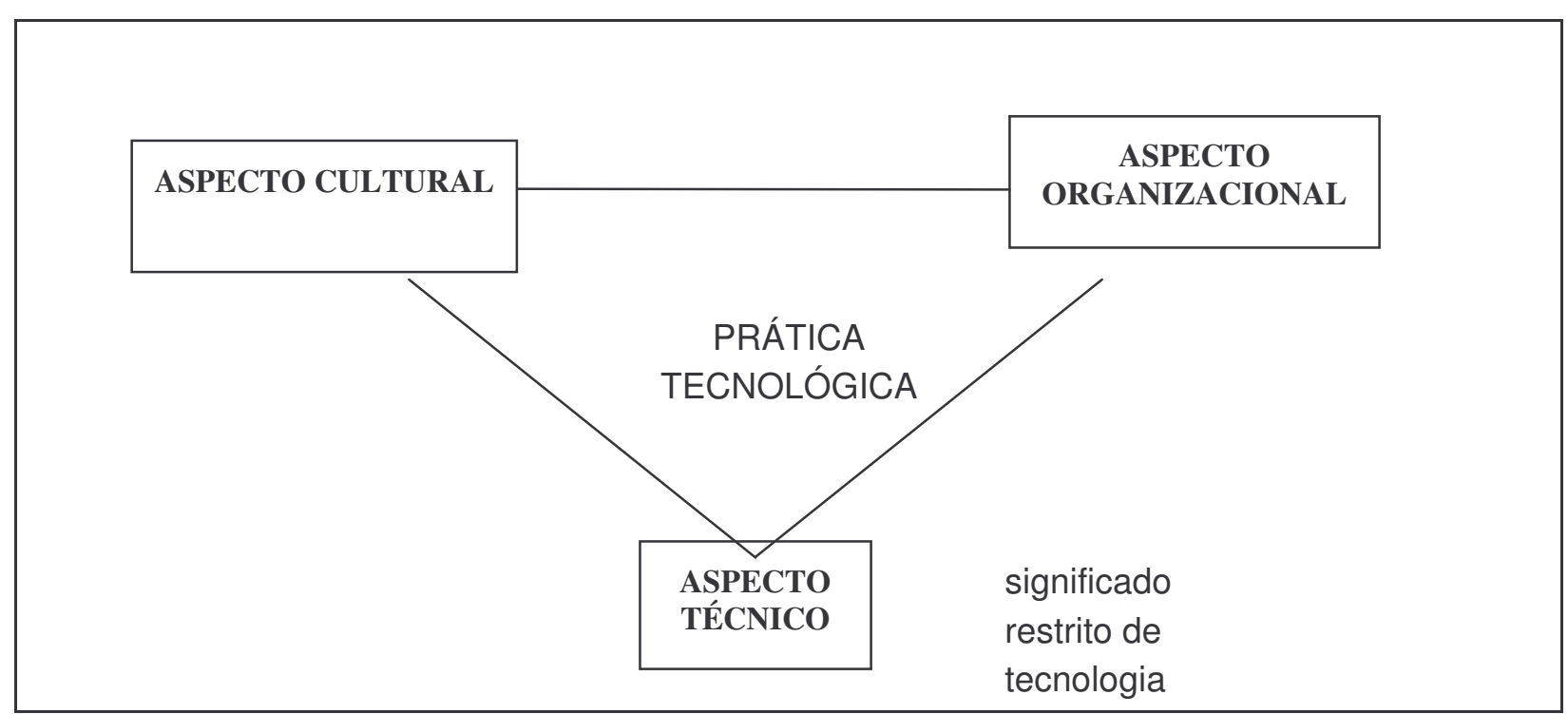

FIGURA 1 - Significado da tecnologia

FONTE - PACEY, 1990. p.19. [tradução nossa]. 
Do diagrama, podemos identificar os seguintes aspectos centrais da prática tecnológica (PACEY, 1990):

1. aspecto técnico: conhecimentos, habilidades e técnicas; instrumentos, ferramentas e máquinas; recursos humanos e materiais; matérias primas, produtos obtidos, dejetos e resíduos;

2. aspecto organizacional: atividade econômica e industrial; atividade profissional dos engenheiros, técnicos e operários da produção; usuários e consumidores; sindicatos;

3. aspecto cultural: objetivos, sistema de valores e códigos éticos, crenças sobre o progresso, consciência e criatividade.

Em geral, a tecnologia é reduzida apenas ao seu aspecto técnico. A identificação dos aspectos organizacionais e culturais da tecnologia permite compreender como ela é dependente dos sistemas sócio-políticos e dos valores e das ideologias da cultura em que se insere. É com esse entendimento que o cidadão passa a perceber as interferências que a tecnologia tem em sua vida e como ele pode interferir nessa atividade.

A alfabetização tecnológica no contexto de CTS inclui a compreensão de todos esses aspectos da prática tecnológica (ACEVEDO, 1996). Segundo FLEMING (1989):

uma pessoa letrada tecnologicamente tem o poder e a liberdade de usar esse poder para examinar e questionar os problemas de importância em sócio-tecnologia. Algumas dessas questões poderiam ser: as idéias de progresso por meio da tecnologia, as tecnologias apropriadas, os benefícios e custos do desenvolvimento tecnológico, os modelos econômicos envolvendo tecnologia, as decisões pessoais envolvendo o consumo de produtos tecnológicos e como as decisões tomadas pelos gerenciadores da tecnologia conformam suas aplicações (: 393-394) [tradução nossa].

Na perspectiva de formar um cidadão que possa compreender como a tecnologia tem influenciado o comportamento humano e desenvolver atitudes em prol de um desenvolvimento tecnológico sustentável, é essencial que haja uma discussão dos valores envolvidos nas decisões (LAYTON, 1988). É a partir da identificação dos valores que se compreendem melhor as necessidades da sociedade e os aspectos éticos que devem ser considerados no uso mais responsável da tecnologia. Será também pelo desenvolvimento de valores que se consolidará o sentimento de solidariedade cósmica, dentro de um novo paradigma em emergência, o da comunidade planetária (BOFF, 1996).

Nesse sentido, entendemos que a educação tecnológica no ensino médio vai muito além do fornecimento de conhecimentos limitados de explicação técnica do funcionamento de determinados artefatos tecnológicos. Não se trata de simplesmente preparar o cidadão para saber lidar com essa ou aquela ferramenta tecnológica ou desenvolver no aluno representações que o instrumentalize a absorver as novas tecnologias. Tais conhecimentos são importantes, mas uma educação que se limite ao uso de novas tecnologias e à compreensão de seu funcionamento é alienante, pois contribui para manter o processo de dominação do homem pelos ideais de lucro a qualquer preço, não contribuindo para a busca de um desenvolvimento sustentável. 


\section{Sociedade}

Os currículos de CTS se articulam em torno de temas científicos ou tecnológicos que são potencialmente problemáticos do ponto de vista social (AIKENHEAD, 1994a; RAMSEY, 1993; RUBBA, 1991; THIER, 1985). Segundo RAMSEY (1993), um tema social relativo à ciência e tecnologia deveria ter sua origem nessas atividades e envolver um problema em torno do qual existam diferentes possibilidades associadas a diferentes conjuntos de crenças e valores.

Nas discussões desses temas, seria importante que fosse evidenciado o poder de influência que os alunos podem ter como cidadãos, bem como as questões éticas e os valores humanos relacionados à ciência e à tecnologia. Dessa maneira, os alunos poderiam ser estimulados a participar democraticamente da sociedade por meio da expressão de suas opiniões (LÓPEZ e CEREZO, 1996; SOLOMON, 1988; RAMSEY, 1993; SOLOMON, 1993b; WAKS, 1990).

Isso poderia ser feito, por exemplo, levando-se os alunos a perceberem o potencial de atuar em grupos sociais organizados, como centros comunitários, escolas, sindicatos, etc. Pode-se mostrar o poder do consumidor em influenciar o mercado, selecionando o que consumir. Além disso, as discussões das questões sociais englobariam os aspectos políticos, os interesses econômicos, os efeitos da mídia no consumo, etc. Questões dessa natureza propiciarão ao aluno uma compreensão melhor dos mecanismos de poder dentro das diversas instâncias sociais.

RAMSEY (1993) apresenta três critérios para identificar um tema social relativo à ciência: (1) se é, de fato, um problema de natureza controvertida, ou seja, se existem opiniões diferentes a seu respeito; (2) se o tema tem significado social e (3) se o tema, em alguma dimensão, é relativo à ciência e à tecnologia.

Alguns autores, como MERRYFIELD (1991), defendem a inclusão, no currículo, de temas globais. Tais temas são caracterizados por afetar a vida das pessoas em várias partes do mundo e por não serem passíveis de compreensão ou tratamento adequado somente em contextos local ou nacional. MERRYFIELD (1991) apresenta os seguintes exemplos de temas globais: (1) temas ambientais; (2) saúde e população; (3) questões econômicas; (4) transporte e comunicação; (5) alimentos e fome; (6) energia e (7) questões militares.

Há divergências quanto à seleção de temas globais ou regionais. Para RAMSEY (1993), a questão central está no grau de problematização social do tema. Ainda que não diretamente relacionadas aos problemas da educação científica e tecnológica, as contribuições de Paulo Freire ajudam a clarear aspectos relativos à discussão dos temas a serem priorizados no currículo. FREIRE (1987) discute que a conscientização do indivíduo ocorre por meio do diálogo com suas condições de existência, o qual se traduz, numa proposta de educação libertadora, por meio do uso de "temas geradores". Os temas, que têm sua origem na situação presente, existencial, concreta dos educandos e refletem as suas aspirações, organizam o conteúdo programático. Como diz FREIRE (1987): "É na realidade mediadora, na consciência que dela tenhamos, educadores e povo, que iremos buscar o conteúdo programático da educação" (: 87). O tema se origina, então, nas relações dos homens com o mundo. A sugestão de FREIRE 
é que se parta de situações locais para a análise de problemas nacionais e regionais (FREIRE, 1996). BOFF (1996), em sua cosmovisão ecológica, considera que: "O bem comum particular emerge a partir da sintonia e sinergia com a dinâmica do bem comum planetário e universal" (: 61). Nesse sentido, poderia ser proposta uma abordagem a partir de problemas locais que se articulassem com a dimensão global.

Os temas, geralmente abordados em cursos de CTS, foram agrupados por TOWSE (1986) nas seguintes áreas: (1) saúde; (2) alimentação e agricultura; (3) recursos energéticos; (4) terra, água e recursos minerais; (5) indústria e tecnologia; (6) ambiente; (7) transferência de informação e tecnologia e (8) ética e responsabilidade social. Já BYBEE (1987) (ver também BYBEE E MAU, 1986) identificou os seguintes temas centrais de cursos CTS: (1) qualidade do ar e atmosfera; (2) fome mundial e fontes de alimentos; (3) guerra tecnológica; (4) crescimento populacional; (5) recursos hídricos; (6) escassez de energia; (7) substâncias perigosas; (8) a saúde humana e doença; (9) uso do solo; (10) reatores nucleares; (11) animais e plantas em extinção e (12) recursos minerais.

No contexto brasileiro, poderiam ser discutidos temas como: (1) exploração mineral e desenvolvimento científico, tecnológico e social. Questões atuais como a exploração mineral por empresas multinacionais, a privatização da Companhia Vale do Rio Doce, as propostas de privatização da Petrobrás, etc. são alguns exemplos de possibilidades nesse tema; (2) ocupação humana e poluição ambiental, na qual seriam discutidos os problemas de ocupação desordenada nos grandes centros urbanos, o saneamento básico, a poluição da atmosfera e dos rios, a saúde pública, a diversidade regional que provoca o êxodo de populações, a questão agrária; (3) o destino do lixo e o impacto sobre o ambiente, o que envolveria reflexões sobre hábitos de consumo na sociedade tecnológica; (4) controle de qualidade dos produtos químicos comercializados, envolvendo os direitos do consumidor, os riscos para a saúde, as estratégias de marketing usadas pelas empresas; (5) a questão da produção de alimentos e a fome que afeta parte significativa da população brasileira, a questão dos alimentos transgênicos; (6) o desenvolvimento da agroindústria e a questão da distribuição de terra no meio rural, custos sociais e ambientais da monocultura; (7) o processo de desenvolvimento industrial brasileiro, a dependência tecnológica num mundo globalizado; nesse tema poderia ser discutida, por exemplo, a exportação de silício bruto ou industrializado; (8) as fontes energéticas no Brasil, seus efeitos ambientais e seus aspectos políticos; (9) a preservação ambiental, as políticas de meio ambiente, o desmatamento. Vários desses temas fazem parte atualmente dos currículos de Geografia. Todavia, dado o forte componente científico e tecnológico deles, é importante que sejam explorados também na área de Ciências e suas Tecnologias, de preferência numa abordagem interdisciplinar junto com a Geografia e outras disciplinas.

\section{Interações ciência-tecnologia-sociedade}

O conteúdo dos currículos de CTS, como acabamos de ver, tem um caráter multidisciplinar (SOLOMON, 1993b). Os conceitos são sempre abordados em uma perspectiva relacional, de maneira a evidenciar as diferentes dimensões do conhecimento estudado, sobretudo as interações entre ciência, tecnologia e sociedade.

Nesses currículos, procura-se evidenciar como os contextos social, cultural e ambiental, 
nos quais se situam a ciência e a tecnologia, influenciam a condução e o conteúdo das mesmas; como ciência e tecnologia, por sua vez, influenciam aqueles contextos e, finalmente, como ciência e tecnologia têm efeitos recíprocos e suas inter-relações variam de época para época e de lugar para lugar (RAMSEY, 1993). Exemplos dessas interações são ilustradas por MCKAVANAGH e MAHER (1982) no QUADRO 1.

\section{QUADRO 1}

\section{Aspectos da abordagem de CTS}

\begin{tabular}{l|l}
\hline \multicolumn{1}{c|}{ Aspectos de CTS } & \multicolumn{1}{c}{ Esclarecimentos } \\
\hline $\begin{array}{l}\text { 1. Efeito da Ciência } \\
\text { sobre a } \\
\text { Tecnologia }\end{array}$ & $\begin{array}{l}\text { A produção de novos conhecimentos tem estimulado mudanças } \\
\text { tecnológicas. }\end{array}$ \\
\hline $\begin{array}{l}\text { 2. Efeito da } \\
\text { Tecnologia sobre } \\
\text { a Sociedade }\end{array}$ & $\begin{array}{l}\text { A tecnologia disponível a um grupo humano influencia } \\
\text { sobremaneira o estilo de vida desse grupo. }\end{array}$ \\
\hline $\begin{array}{l}\text { 3. Efeito da } \\
\text { Sociedade sobre a }\end{array}$ & $\begin{array}{l}\text { Por meio de investimentos e outras pressões, a sociedade } \\
\text { influencia a direção da pesquisa científica. }\end{array}$ \\
\hline $\begin{array}{l}\text { 4. Efeito da Ciência } \\
\text { sobre a Sociedade }\end{array}$ & $\begin{array}{l}\text { O desenvolvimento de teorias científicas podem influenciar a } \\
\text { maneira como as pessoas pensam sobre si próprias e sobre } \\
\text { problemas e soluções. }\end{array}$ \\
\hline $\begin{array}{l}\text { Efeito da } \\
\text { Sociedade sobre a } \\
\text { Tecnologia }\end{array}$ & $\begin{array}{l}\text { Pressões públicas e privadas podem influenciar a direção em que } \\
\text { os problemas são resolvidos e, em conseqüência, promover } \\
\text { mudanças tecnológicas. }\end{array}$ \\
\hline $\begin{array}{l}\text { 6. Efeito da } \\
\text { Tecnologia sobre } \\
\text { a Ciência }\end{array}$ & $\begin{array}{l}\text { A disponibilidade dos recursos tecnológicos limitará ou ampliará os } \\
\text { progressos científicos. }\end{array}$ \\
\hline
\end{tabular}

FONTE - McKAVANAGH e MAHER, 1982. p.72. [tradução nossa].

Um estudo das aplicações da ciência e tecnologia, sem explorar as suas dimensões sociais, podem propiciar uma falsa ilusão de que o aluno compreende o que é ciência e tecnologia. Esse tipo de abordagem pode gerar uma visão deturpada sobre a natureza desses conhecimentos, como se estivessem inteiramente a serviço do bem da humanidade, escondendo e defendendo, mesmo que sem intenção, os interesses econômicos daqueles que desejam manter o status quo.

\section{c) Estratégias de ensino}

Pesquisas sobre abordagens mais efetivas de CTS geralmente indicam que os seus materiais de ensino são melhores organizados na seqüência de etapas sugeridas na FIG. 2 (AIKENHEAD, 1994a). A seta da figura indica que a estrutura dos materiais de ensino de CTS é seqüenciada pelos passos: (1) introdução de um problema social; (2) análise da tecnologia relacionada ao tema social; (3) estudo do conteúdo científico definido em função do tema social 
e da tecnologia introduzida; (4) estudo da tecnologia correlata em função do conteúdo apresentado e (5) discussão da questão social original.

No livro Química na Sociedade (MÓL e SANTOS, 2000), a abordagem do conteúdo químico é feita por meio de temas sociais. Esse material didático introduz o conteúdo a partir de um texto gerador, que apresenta um tema de relevância social, problematizando-o e estabelecendo relações com determinados conceitos químicos, que serão necessários para sua abordagem. Em seguida, esses conceitos são apresentados ao aluno e, após essa fase, são explorados textos que retomam o tema em foco na unidade, para explicar as relações entre essa e os conceitos químicos estudados. Tais textos apontam a necessidade de estudo de novos conceitos e assim sucessivamente, em um modelo curricular em espiral que permite que o conteúdo programático proposto seja esgotado. Ao final, as dimensões sociais do tema são novamente postas em evidência e uma série de atividades relacionadas à tomada de decisão são introduzidas, as quais exploram os aspectos ambientais, políticos, econômicos, éticos, sociais e culturais.

O estudo de temas, por meio da seqüência ilustrada acima, permite a introdução de problemas sociais a serem discutidos pelos alunos, propiciando o desenvolvimento da capacidade de tomada de decisão. Para isso, a abordagem dos temas é feita por meio da introdução de problemas, cujas possíveis soluções são propostas em sala de aula após a discussão de diversas alternativas, surgidas a partir do estudo do conteúdo científico, de suas aplicações tecnológicas e conseqüências sociais.

Ainda nessa perspectiva, são sugeridas diversas atividades para o ensino de CTS. HOFSTEIN, AIKENHEAD e RIQUARTS (1988) apontam, entre outras, as seguintes estratégias utilizadas em CTS: palestras, demonstrações, sessões de discussão, solução de problemas, jogos de simulação e desempenho de papéis, fóruns e debates, projetos individuais e de grupo, redação de cartas a autoridades, pesquisa de campo e ação comunitária.

AIKENHEAD (1994b) e SOLOMON (1993a) relacionam as seguintes atividades geralmente adotadas no ensino de CTS: pensamento divergente, solução de problema, simulações, atividades de tomada de decisão, controvérsias, debates. Essas atividades seriam realizadas por meio de trabalho em pequenos grupos, discussão em sala de aula centrada nos estudantes, e poderiam envolver o uso de recursos da mídia e outras fontes comunitárias.

Outras atividades recomendadas são estudo de caso, envolvendo problemas reais da sociedade (BYRNE e JOHNSTONE, 1988; HEATH, 1992; LAETER e LUNETTA, 1982), construção de modelos de artefatos tecnológicos (JAMIESON, MILLER e WATTS, 1993; SOLOMOM, 1993b), uso de fatos da história da ciência e discussão em grupo sobre vídeos envolvendo questões científicas e tecnológicas (SOLOMON, 1989, 1993b). Essas atividades são geralmente sugeridas na forma de trabalhos cooperativos entre alunos ou entre professor $\mathrm{e}$ aluno, pesquisas, apresentações orais e relatórios escritos (HEATH, 1992).

Todas essas sugestões metodológicas contribuem para que os alunos desenvolvam habilidades e atitudes necessárias à tomada de decisão. Sobre tal objetivo, vários autores apresentam modelos e roteiros de atividades para propiciar o seu desenvolvimento. Esses modelos geralmente caracterizam-se por uma proposta racional de análise de custos e benefícios, feita a partir de uma seqüência de passos normativos (KORTLAND, 1996; McCONELL, 1982; RATCLIFFE, 1997). 
Essa forma racionalista de encarar a tomada de decisão, muitas vezes envolvendo a escolha dicotômica entre uma ou outra alternativa, é passível de crítica, pois muitos dos problemas presentes no contexto social do aluno envolvem não a escolha entre, mas a superação de, alternativas dicotômicas por meio de sínteses dialéticas.

Segundo HABERMAS (1973), as decisões sobre as interações entre a ciência e tecnologia e a sociedade podem ser tomadas de acordo com os modelos tecnocráticos, decisionistas e pragmático-políticos. No modelo tecnocrático, a decisão política é tomada exclusivamente em função do referencial dos especialistas em ciências e em tecnologia. No modelo decisionista, os cidadãos determinam os fins, os meios e os técnicos que vão participar da decisão, mas essa é tomada pelo especialista, segundo os critérios estabelecidos. Já no modelo pragmático-político, há uma interação e negociação entre os especialistas e os cidadãos.

Alguns trabalhos apontam essa questão da tomada de decisão como um dilema dos currículos de CTS, pois a análise desse processo é por demais complexa (SOLOMON, 1991 e 1994). Nesse sentido, é preciso refletir sobre os diversos fatores que influenciam a atitude dos estudantes frente a um problema social, o que não pode ser reduzido à mera análise da interação do aluno com o material de CTS. É preciso, ainda, discutir a relação problemática entre atitudes desenvolvidas na escola e ação social subseqüente, pois aparentemente não há uma correspondência direta unilateral entre as atitudes desenvolvidas nos cursos de CTS e a participação dos alunos em questões sociais na vida diária (LAYTON, 1994; SOLOMON, 1991 e 1994; THOMAS, 1985).

Além disso, estudos sobre a natureza do conhecimento científico e suas relações com o conhecimento humano em geral (JENKINS, no prelo) mostram que a ciência com que as pessoas lidam na vida real raramente é objetiva, coerente, bem delimitada e não problemática. E que o conhecimento científico, longe de ser central para muitas das decisões sobre ações práticas, é irrelevante ou, quando muito, marginal em relação a essas decisões. Essas considerações precisam ser aprofundadas na discussão sobre currículos CTS, pois corre-se o risco de se estabelecer uma relação artificial entre conhecimento científico e resolução de problemas, que não corresponde à realidade. Por exemplo, a maioria dos problemas que técnicos e engenheiros enfrentam relativos a processos de transferência de calor ou isolamento térmico de ambientes não são tratados a partir de um modelo cinético-molecular de calor, mas pelo uso de um modelo de calor como fluido, à semelhança da idéia de calórico. A existência de uma diversidade de modelos alternativos para os mesmos fenômenos, de um perfil conceitual (MORTIMER, 1995 e 1998) para cada conceito científico, força-nos a reconhecer que a questão do uso de conceitos científicos na sociedade está longe de ser direta e não problemática. Os conceitos cotidianos continuarão a ter peso na maioria das decisões que tomamos na vida cotidiana, independente de nosso conhecimento de alternativas mais científicas, pois, muitas vezes, essas são inúteis para a solução desses problemas. O reconhecimento desses limites evita a ilusão, que no fundo é cientificista, de que a ciência poderá, num futuro, informar todas as nossas decisões. 


\section{Categorias curriculares}

Nem todas as propostas de ensino que vêm sendo denominadas CTS estão centradas nas inter-relações entre ciência, tecnologia e sociedade. Isso tem levado ao estabelecimento de várias classificações desses cursos, conforme o foco central (AIKENHEAD, 1990; AIKENHEAD, 1994a; FENSHAM, 1988; GASKELL, 1982; LOWE, 1985; ROSENTHAL, 1989).

A diferença entre as categorias para agrupar os cursos é função da prioridade que tem sido atribuída para cada um dos objetivos gerais de CTS e da proporção entre o conteúdo de CTS e o conteúdo puro de ciências. A classificação de AIKENHEAD (1994a), apresentada no QUADRO 2, ilustra essas diferenças. À medida que se progride nas categorias, a avaliação de conteúdos CTS aumenta progressivamente em relação à avaliação do conteúdo puro de ciências. Nessa escala, a categoria 1 corresponderia a $0 \%$ de avaliação de conteúdos CTS e a categoria 8 a $100 \%$.

\section{QUADRO 2}

Categorias de ensino de CTS

\begin{tabular}{|c|c|}
\hline Cat & ão \\
\hline $\begin{array}{l}\text { Conteúdo } \\
\text { le CTS como } \\
\text { lemento de } \\
\text { notivação. }\end{array}$ & $\begin{array}{l}\text { Ensino tradicional de ciências O que muitos profes } \\
\text { acrescido da menção ao "dourar a pílula" de } \\
\text { conteúdo de CTS com a função conceituais } \\
\text { de tornar as aulas mais } \\
\text { interessantes. }\end{array}$ \\
\hline $\begin{array}{l}\text { ncorporação } \\
\text { eventual do } \\
\text { conteúdo de } \\
\text { CTS ao } \\
\text { conteúdo } \\
\text { orogramático. }\end{array}$ & $\begin{array}{l}\text { Ensino tradicional de ciências Science and Technology in Society } \\
\text { acrescido de pequenos estudos (SATIS, UK), Consumer Science (EUA), } \\
\text { de conteúdo de CTS Values in School Science (EUA). } \\
\text { incorporados como apêndices } \\
\text { aos tópicos de ciências. O } \\
\text { conteúdo de CTS não é } \\
\text { resultado do uso de temas } \\
\text { unificadores. }\end{array}$ \\
\hline $\begin{array}{l}3 . \\
\text { Incorporação } \\
\text { sistemática do } \\
\text { conteúdo de } \\
\text { CTS ao } \\
\text { conteúdo } \\
\text { orogramático. }\end{array}$ & $\begin{array}{l}\text { Ensino tradicional de ciências Havard Project Physics (EUA), Science } \\
\text { acrescido de uma série de and Social Issues (EUA), Nelson } \\
\text { pequenos estudos de conteúdo Chemistry (Canadá), Interactive } \\
\text { de CTS integrados aos tópicos Teaching Units for Chemistry (UK), } \\
\text { de ciências, com a função de Science, Technology and Society, Block } \\
\text { explorar sistematicamente o J. (EUA). Three SATIS 16-19 modules } \\
\text { conteúdo de CTS. Esses (What is Science? What is Technology? } \\
\text { conteúdos formam temas How Does Society decide?- UK). } \\
\text { unificadores. }\end{array}$ \\
\hline
\end{tabular}

4. Disciplina Os temas de CTS são utilizados ChemCon (EUA), os módulos científica para organizar o conteúdo de holandeses de física como Light Sources 


\begin{tabular}{|c|c|c|}
\hline $\begin{array}{l}\text { Zuímica, } \\
\text { ísica } \\
\text { iologia) } \\
\text { eio } \\
\text { onteúdo } \\
\text { TS }\end{array}$ & $\begin{array}{l}\text { ciências e a sua seqüência, mas } \\
\text { a seleção do conteúdo científico } \\
\text { ainda é a feita partir de uma } \\
\text { disciplina. A lista dos tópicos } \\
\text { científicos puros é muito } \\
\text { semelhante àquela da categoria } \\
\text { 3, embora a seqüência possa ser } \\
\text { bem diferente. }\end{array}$ & $\begin{array}{l}\text { and lonizing Radiation (Holanda: PLON), } \\
\text { Science and Society Teaching units } \\
\text { (Canadá), Chemical Education for Public } \\
\text { Understandig (EUA), Science Teachers' } \\
\text { Association of victoira Physics Series } \\
\text { (Austrália). }\end{array}$ \\
\hline \begin{tabular}{lr}
\multicolumn{2}{c}{ Ciências por } \\
eio do \\
onteúdo de \\
TS
\end{tabular} & $\begin{array}{l}\text { CTS organiza o conteúdo e sua } \\
\text { seqüência. O conteúdo de } \\
\text { ciências é multidisciplinar, sendo } \\
\text { ditado pelo conteúdo de CTS. A } \\
\text { lista de tópicos científicos puros } \\
\text { assemelha-se à listagem de } \\
\text { tópicos importantes a partir de } \\
\text { uma variedade de cursos de } \\
\text { ensino tradicional de ciências. }\end{array}$ & $\begin{array}{l}\text { Logical Reasoning in Science and } \\
\text { Technology (Canadá), Modular STS } \\
\text { (EUA), Global Science (EUA), Dutch } \\
\text { Environmental Project (Holanda), Salters' } \\
\text { Science Project (UK) }\end{array}$ \\
\hline $\begin{array}{l}\text { Ci } \\
\text { som con } \\
\text { le CTS }\end{array}$ & $\begin{array}{l}\text { udo de CTS é o foco do } \\
\text { conteúdo relevante de } \\
\text { enriquece a } \\
\text { agem. }\end{array}$ & $\begin{array}{l}\text { Exploring the Nature of Science (Ing.) } \\
\text { Society Environment and Energy } \\
\text { Development Studies (SEEDS) modules } \\
\text { (EUA), Science and Technology } 11 \\
\text { (Canadá) }\end{array}$ \\
\hline $\begin{array}{l}7 . \\
\text { Incorporação } \\
\text { das Ciências } \\
\text { ao conteúdo } \\
\text { de CTS }\end{array}$ & $\begin{array}{l}\text { O conteúdo de CTS é o foco do } \\
\text { currículo. O conteúdo relevante } \\
\text { de ciências é mencionado, mas } \\
\text { não érinado } \\
\text { sistematicamente. Pode ser } \\
\text { dada ênfase aos princípios } \\
\text { gerais da ciência. }\end{array}$ & $\begin{array}{l}\text { Studies in a Social Context (SISCON) in } \\
\text { Schools (UK), Modular Courses in } \\
\text { Technology (UK), Science A Way of } \\
\text { Knowning (Canadá), Science } \\
\text { Technology and Society (Austrália), } \\
\text { Creative Role Playing Exercises in } \\
\text { Science and Technology (EUA), Issues } \\
\text { for Today (Canadá), Interactions in } \\
\text { Science and Society - vídeos (EUA), } \\
\text { Perspectives in Science (Canadá) }\end{array}$ \\
\hline $\begin{array}{l}\text { 8. Conteúdo } \\
\text { de CTS }\end{array}$ & $\begin{array}{l}\text { Estudo de uma questão } \\
\text { tecnológica ou social importante. } \\
\text { O conteúdo de ciências é } \\
\text { mencionado somente para } \\
\text { indicar uma vinculação com as } \\
\text { ciências. }\end{array}$ & $\begin{array}{l}\text { Science and Society (UK.), Innovations: } \\
\text { The Social Consequencies of Science } \\
\text { and Technology program (EUA), } \\
\text { Preparing for Tomorrow's World (EUA), } \\
\text { Values and Biology (EUA). }\end{array}$ \\
\hline
\end{tabular}

FONTE - AIKENHEAD, 1994a. p. 55-56. [tradução nossa].

AIKENHEAD (1994a) considera que, embora nenhuma das categorias possa representar o modelo "real" de CTS, as categorias de 3 a 6 são as que representam a visão 
mais comumente citada na literatura. Um curso classificado na categoria 1 talvez nem pudesse ser considerado como CTS, dado o baixo status atribuído ao conteúdo de CTS. Já a categoria 8 refere-se a cursos radicais de CTS, em que os conteúdos de ciências propriamente ditos praticamente não são abordados. Percebe-se, assim, que até a categoria 4 há uma maior ênfase no ensino conceitual de ciências e, a partir da categoria 5, a ênfase muda para a compreensão dos aspectos das inter-relações de CTS.

Currículos nas categorias 6 e 7 poderiam ser propostos dentro da atual reforma do ensino médio, na tentativa de se buscar a interdisciplinaridade na área de ciências da natureza e suas tecnologias. Obviamente que tal proposição demandaria projetos audaciosos a serem desenvolvidos com a participação de professores, o que não poderia ser feito de maneira aleatória.

\section{Considerações finais}

O processo de implantação de currículos de CTS vem ocorrendo em diversos países desde a década de setenta, com a elaboração de materiais didáticos, sua aplicação e avaliação e a realização de cursos de formação de professores (SOLOMON e AIKENHEAD, 1994). Esse processo de implantação tem sido avaliado por inúmeras pesquisas, as quais têm constatado que os estudantes, de uma maneira geral, têm se beneficiado com a introdução desses currículos (AIKENHEAD, 1994b).

Acreditamos que, da mesma forma, projetos curriculares nacionais poderão ser desenvolvidos com ênfase em CTS, à semelhança de algumas propostas que foram citadas anteriormente (LUTFI 1988 e 1992; GEPEQ, 1993, 1995, 1998; GREF, 1990, 1991 e 1993; MÓL e SANTOS, 2000; MORTIMER, MACHADO e ROMANELLI, 1998 e 1999). O contexto atual é bastante favorável para a elaboração de projetos nacionais de ensino de ciências, tanto para o ensino fundamental como para o médio, com ênfase em CTS. Entendemos que tais currículos muito podem contribuir para a alfabetização e o letramento científico e tecnológico, pois alfabetizar é, como propunha Paulo Freire, um ato de conscientização política.

Mas, para isso, uma série de questionamentos precisam ser levantados: Que cidadãos se pretende formar por meio das propostas CTS? Será o cidadão no modelo capitalista atual, pronto a consumir cada vez mais, independente do reflexo que esse consumo tenha sobre o ambiente e sobre a qualidade de vida da maioria da população? Que modelo de tecnologia desejamos: clássica ecodesequilibradora ou de desenvolvimento sustentável? O que seria um modelo de desenvolvimento sustentável? Que modelo decisionista desenvolveremos no nosso aluno, o tecnocrático ou o pragmático-político?

É preciso compreender, também, o contexto dos países em que as propostas curriculares de CTS foram desenvolvidas. Por se tratar de países desenvolvidos, a estrutura social, a organização política e o desenvolvimento econômico são bastante diferentes daqueles presentes no contexto brasileiro. Isso implica que seria um contra-senso a transferência acrítica de modelos curriculares desses países para o nosso meio educacional. Problemas relacionados às desigualdades sociais extremas, por exemplo, não existem nos países em que esses currículos foram desenvolvidos. Discutir modelos de currículos de CTS significa, portanto, 
discutir concepções de cidadania, modelo de sociedade, de desenvolvimento tecnológico, sempre tendo em vista a situação sócio-econômica e os aspectos culturais do nosso país.

Por outro lado, a revisão da literatura internacional nos ajuda a ver que adotar propostas CTS é muito diferente de simplesmente maquiar currículos com ilustrações do cotidiano. Currículos de CTS diferenciam-se significativamente dos currículos convencionais (RUBBA, 1991; RAMSEY, 1993; YAGER, 1990; YAGER e TAMIR, 1993; ZOLLER e WATSON, 1974). Assim, as mudanças a serem efetivadas são muito mais profundas do que a mera adoção de temas (LÓPEZ e CEREZO, 1996). Os princípios diferenciadores são vários: a preocupação com a formação de atitudes e valores em contraposição ao ensino memorístico de pseudopreparação para o vestibular; a abordagem temática em contraposição aos extensos programas de ciências alheios ao cotidiano do aluno; o ensino que leve o aluno a participar em contraposição ao ensino passivo, imposto sem que haja espaço para a sua voz e suas aspirações. Enfim, uma reforma curricular de CTS implica mudanças de concepções do papel da educação e do ensino das ciências.

Temos que considerar, também, que diversas pesquisas de avaliações de currículos de CTS têm apontado para a necessidade de acompanhamento do processo de implantação curricular no que diz respeito à formação de professores (BYBEE, 1987; BYBEE e MAU, 1986; FLEMING, 1988; GASKELL, 1982; HART e ROBOTTOM, 1990; HOFSTEIN, AIKENHEAD E RIQUARTS, 1988; LOWE, 1985; MITCHENER E ANDERSON, 1989; RUBBA, 1989; WAKS e BARCHI, 1992; ZOLLER, DONN, WILD e BECKETT 1991a, b).

Nesse sentido, acreditamos que a proposta de HART e ROBOTTOM (1990), a seguir, aponta para um caminho que poderia ser trilhado:

O processo da reforma na educação em ciências deverá ser elaborado de modo a criar condições para que os próprios praticantes reflitam criticamente, deliberem de maneira colaborativa e se engajem em pesquisa participante sobre os potenciais e os limites das propostas de reforma CTS para a educação em ciências. Assim como os alunos devem ser envolvidos na tomada de decisões sociais relacionadas à ciência e à tecnologia, também os professores devem ser envolvidos na tomada de decisões sobre a educação em ciências (HART e ROBOTTOM, 1990: 585) [tradução nossa].

Isso evidencia que a reforma curricular atual do ensino médio depende de um processo de formação continuada de professores para que não se torne letra morta na legislação. Como desenvolver novos modelos curriculares sem envolver aqueles que irão aplicar tais modelos? Não adianta apenas inserir temas sociais no currículo, sem qualquer mudança significativa na prática e nas concepções pedagógicas. Não basta as editoras de livros didáticos incluírem em seus livros temas sociais, ou disseminarem os chamados paradidáticos. Sem uma compreensão do papel social do ensino de ciências, podemos incorrer no erro da simples maquiagem dos currículos atuais com pitadas de aplicação das ciências à sociedade. Ou seja, sem contextualizar a situação atual do sistema educacional brasileiro, das condições de trabalho e de formação do professor, dificilmente poderemos contextualizar os conteúdos científicos na perspectiva de formação da cidadania. 


\section{Referências}

ACEVEDO DÍAZ, J. A. (1996). La tecnología en las relaciones CTS: una aproximación al tema. Enseñanza de las Ciencias, v.14, n.1, p.35-44.

AIKENHEAD, G. S. (1985). Collective decision making in the social context of science. Science Education, v. 69, n. 4.

. (1990). Science-technology-society Science education development: from curriculum policy to student learning. Brasília: CONFERÊNCIA INTERNACIONAL SOBRE ENSINO DE CIÊNCIAS PARA O SÉCULO XXI: ACT - Alfabetização em ciência e tecnologia, 1; jun/1990. (Mimeogr.).

. (1994a). What is STS science teaching? In: SOLOMON, J., AIKENHEAD, G. STS education: international perspectives on reform. New York: Teachers College Press, p.47-59. (1994b). Consequences to learning science through STS: a research perspective In: SOLOMON, J., AIKENHEAD, G. STS education: international perspectives on reform. New York: Teachers College Press, p.169-186.

ALVES, R. (1968). Tecnologia e humanização. In: Revista Paz e Terra, II, n.8.

AMBROGI, A. et al. (1987). Unidades modulares de química. São Paulo: Hamburg.

BAZZO, W. A. (1998). Ciência, tecnologia e sociedade: e o contexto da educação tecnológica. Florianópolis: EDUFSC.

BERNARD, F., CROMMELINCK M. (1992). Sciences de la nature, technologies et sociétés. In: MEULDERS, M., CROMMELINCK, M., FELTZ B. Pourquoi la science? Paris: Champ Vallon.

BOFF, L. (1996). Ecologia: grito da terra, grito dos pobres, 2.ed. São Paulo: Ática.

BRIDGSTOCK, M. et al. (1998). Science, technology and society: an introduction. Australia: Cambridge University Press.

BYBEE, R. W. (1987). Science education and the science-technology-society (STS) theme. Science Education, v. 71, n. 5, p.667-683.

BYBEE, R. W., MAU, T. (1986). Science and technology related global problems: an international survey of science educators. Journal of Research in Science Teaching, v. 23, n. 7, p.599-618.

BYRNE, M. S., JOHNSTONE, A. H. (1988). How to make science relevant. School Science Review, v. 70, n. 251, p.43-46.

CHALMERS, A. F. (1994). A fabricação da ciência. São Paulo: Editora da Universidade Estadual Paulista.

CISCATO, C. A. M., BELTRAN, N. O. (1991). Química: parte integrante do projeto diretrizes gerais para o ensino de $2^{\circ}$ grau núcleo comum (convênio MEC/PUC-SP). São Paulo: Cortez e Autores Associados.

DRIVER, R., ASOKO, H., LEACH, J., MORTIMER, SCOTT, P. (1994). Constructing Scientific knowledge in the classroom. Educational Researcher, v.23, n.7, p.5-12.

FENSHAM, P. J. (1988). Approaches to the teaching of STS in science education. International Journal of Science education, v. 10, n. 4, p.346-356.

FLEMING, R. (1988). Undergraduate science students' views on the relationship between science, technology and society. International Journal of Science Education, v.10, n.4, p.449463. 
. (1989). Literacy for a technological age. Science Education, v. 73, n. 4, p.391-404.

FOUREZ, G. (1995). A construção das ciências: introdução à filosofia e à ética das ciências. São Paulo: Editora da Universidade Estadual Paulista.

FREIRE, P. (1987). Pedagogia do oprimido, 17.ed. Rio de Janeiro: Paz e Terra. . (1996). Educação como prática da liberdade. 22.ed. Rio de Janeiro: Paz e Terra.

GASKELL, P. J. (1982). Science education for citizens: perspectives and issues. I. Science, technology and society: Issues for science teachers. Studies in Science Education, n. 9, p.33-46.

GEPEQ - Grupo de Pesquisa para o Ensino de Química. (1993/1995/1998). Interação e transformação: química para o $2^{\circ}$ grau. São Paulo: EDUSP. Vol. I, II e III. (livro do aluno: guia do professor).

GIBBONS, M. et al. (1994). The new production of knowledge: the dynamics of science and research in contemporary societies. London: SAGE Publications.

GREF - Grupo de Reelaboração do Ensino de Física. (1990). Física 1: mecânica. São Paulo: EDUSP.

(1991). Física 2: física térmica; óptica. São Paulo: EDUSP.

(1993). Física 3: eletromagnetismo. São Paulo: EDUSP.

HABERMAS, J. (1973). La science et la technique comme "idéologie". Paris: Gallimard.

. (1983). Técnica e ciência enquanto "ideologia". In: BENJAMIN, W., HORKHEIMER, M., ADORNO, T.W., HABERMAS, J. Textos escolhidos. 2.ed. São Paulo: Abril Cultural.

HART, E. P., ROBOTTOM, I. M. (1990). The science-technology-society movement in science education: a critique of the reform process. Journal of Research in Science Teaching, v. 27, n. 6, p.575-588.

HEATH, P. A. (1992). Organising for STS teaching and learning: the doing of STS. Theory into Practice, v. 31, n. 1, p.52-58.

HOFSTEIN, A., AIKENHEAD, G., RIQUARTS, K. (1988). Discussions over STS at the fourth IOSTE symposium. International Journal of Science Education, v. 10, n. 4, p.357-366.

HOLMAN, J. (1988). Editor's introduction: Science-technology-society education. International Journal of Science Education, v. 10, n. 4, p.343-345.

IGLESIA, P. M. (1995). Ciencia-Tecnología-Sociedad en la enseñanza-aprendizaje de las ciencias experimentales. Alambique dicáctica de las ciencias experimentales, v. 2, n. 3, p.711.

JAMIESON, I., MILLER, A., WATTS, A. G. (1993). Productions simulations. In: McCORMICK, R., MURPHY, P., HARRISON, M. (Eds.). Teaching and learning technology. Workingham: Addison-Wesley Publishing Company \& The Open University. p.251-267.

JAPIASSU, H. (1999). Um desafio à educação: repensar a pedagofia científica. São Paulo: Letras \& Letras.

JENKINS, E. Science for All: Time for a Paradigm Shift? In: LEACH, J., MILLAR, R., OSBORNE, J. (Eds.). Improving Science Education: The Contribution of Research: a book in honour of Rosalind Driver. Milton Keynes: Open University Press. (no prelo).

KORTLAND K. (1996). An STS case study about students' decision making on the waste issue. Science Education, v. 80, n. 6, p.673-689.

KRASILCHIK, M. (1987). O professor e o currículo das ciências. São Paulo: EDUSP. 
LAETER, J. R., LUNETTA, V. N. (1982). Environmental issues: a responsibility of science teachers. The Australian Science Teachers Journal, v. 28, n. 3, p.5-10.

LATOUR, B., WOOLGAR, S. (1997). A vida de laboratório: a produção dos fatos científicos. Rio de Janeiro: Relume Dumará.

LAYTON, D. (1988). Revaluing the T in STS. International Journal of Science Education, v. 10, n. 4, p.367-378.

. (1994). STS in the school curriculum: a movement overtaken by history? In: SOLOMON, J., AIKENHEAD, Glen. STS education: international perspectives on reform. New York: Teachers College Press. p.32-44.

LAYTON, D., DAVEY, A., JENKINS, E. (1986). Science for specific social purposes (SSSP): perspectives on adult scientific literacy. Studies in Science Education, n. 13, p.27-52.

LÓPEZ, J. L. L., CEREZO, J. A. L. (1996). Educación CTS en acción: enseñanza secundaria y universidad. In: GARCÍA, M. I. G., CEREZO, J. A. L., LÓPEZ, J. L. L. Ciencia, tecnología y sociedad: una introducción al estudio social de la ciencia y la tecnología. Madrid: Editorial Tecnos S. A.

LOWE, I. (1985). STS: The future mode of science education. The Australian Science Teachers Journal, v. 31, n. 1, p.23-32.

LUTFI, M. (1988). Cotidiano e educação em química: os aditivos em alimentos como proposta para o ensino de química no segundo grau. ljuí: UNIJUÍ.

(1992). Os ferrados e os cromados: produção social e apropriação privada do conhecimento químico. Ijuí: UNIJUÍ.

McCONNELL, M. C. (1982). Teaching about science, technology and society at the secondary school level in the United States: an education dilemma for the 1980s. Studies in Science Education, n. 9, p.1-32.

McKAVANAGH, C., MAHER, M. (1982). Challenges to science education and the STS response. The Australian Science Teachers Journal, v. 28, n. 2, p.69-73.

MERRYFIELD, M. M. (1991). Science-technology-society and global perspectives. Theory into Practice, v. 30, n. 4, p.288-293.

MITCHENER, C. P., ANDERSON, R. D. (1989). Teachers' perspective: developing and implementing an STS curriculum. Journal of Research in Science Teaching, v. 26, n. 4, p.351-369.

MÓL, G. de S.; e SANTOS, W. L. P. dos. (Coords.). (2000). Química na sociedade. 2 ed. Brasília: Editora da UnB.

MORAIS, J. F. R. (1988). Filosofia da ciência e da tecnologia: introdução metodológica e crítica. 5.ed. Campinas: Papirus.

MORTIMER, E. F. (1995). Conceptual change or conceptual profile change? Science \& Education, n.4, p.267-285.

(1998). Multivoicedness and univocality in the classroom discourse: an example from theory of matter. International Journal of Science Education, v. 20, n. 1, p.67-82.

MORTIMER, E. F., MACHADO, A. H., ROMANELLI, L. I. (1998). Proposta curricular - Química: fundamentos teóricos. Belo Horizonte: Secretaria de Estado da Educação de Minas Gerais.

MORTIMER, E. F., MACHADO, A. H., ROMANELLI, L. I. (1999). Química, Energia e ambiente. Belo Horizonte: CECIMIG. 
PACEY, A. (1990). La cultura de la tecnología. Cidade do México: Fondo de Cultura Económica. RAMSEY, J. (1993). The science education reform movement: implications for social responsibility. Science Education, v. 77, n. 2, p.235-258.

RATCLIFFE, M. (1997). Pupil decision-making about socio-scientific issues within the science curriculum. International Journal of Science Education, v. 19, n. 2, p.167-182.

ROBERTS, D. A (1991). What counts as science education? In: FENSHAM, P., J. (Ed.) Development and dilemmas in science education. Barcombe: The Falmer Press, p.27-55.

ROSENTHAL, D. B. (1989). Two approaches to science - technology - society (STS) education. Science Education, v. 73, n. 5, p.581-589.

RUBBA, P. A. (1989). An investigation of the semantic meaning assigned to concepts affiliated with STS education and of STS instructional practices among a sample of exemplary science teachers. Journal of Research in Science Teaching, v. 26, n. 8, p.687-702.

. (1991). Integration STS into school science and teacher education: beyond awareness. Theory into Practice, v. 30, n. 4, p.303-315.

RUBBA, P. A., WIESENMAYER, R. L. (1988). Goals and competencies for precollege STS education: recommendations based upon recent literature in environmental education. Journal of environmental Education, v. 19, n. 4, p.38-44.

SANTOS, W. L. P., SCHNETZLER, R. P. (1997). Educação em química: compromisso com a cidadania. ljuí: UNIJUÍ.

SÃO PAULO. Secretaria de Estado da Educação. Coordenadoria de Estudos e Normas Pedagógicas. (1988). Proposta curricular para o ensino de química: $2^{\circ}$ grau. 2.ed. São Paulo: SE/CENP.

SOLOMON, J. (1988). Science technology and society courses: Tools for thinking about social issues. International Journal of Science Education, v. 10, n. 4, p.379-387.

(1989). The social construction of school science. In: MILLAR, R. (Ed.) Doing science: images of science in science education. London, New York, Philadelphia: The Falmer Press, p.126-136.

(1991). The dilemma of science, technology and society education. In: FENSHAM, P. J. (Ed.) Development and dilemmas in science education. Barcombe: The Falmer Press, p.266281.

. (1993a). Methods of teaching STS. In: McCORMICK, R., MURPHY, P., HARRISON, M. (Eds.). Teaching and learning technology. Workingham: Addison-Wesley Publishing Company \& The Open University, p.243-250

. (1993b). Teaching science, technology and society. Buckingham: Open University Press. . (1994). Toward a map of problems in STS research. In: SOLOMON, J., AIKENHEAD, G. STS education: international perspectives on reform. New York: Teachers College Press, p.187-193.

SOLOMON, J., AIKENHEAD, G. (1994). STS education: international perspectives on reform. New York: Teachers College Press.

STIEFEL, B. M. (1995). La naturaleza de la ciencia en los enfoques CTS. Alambique dicáctica de las ciencias experimentales, v. 2, n. 3, p.19-29.

THIER, H. D. (1985). Societal issues and concerns a new emphasis for science education. Science Education, v. 69, n. 2, p.155-162. 
THOMAS, I. D. (1985). Assessing student understanding of science - technology - society interactions. The Australian Science Teachers Journal, v. 31, n. 1, p.33-37.

TOWSE, P. J. (1986). Editorial. International Newsletter on Chemical Education - IUPAC, n. 2, p.2-3. (Tradução de: International Newsletter on Chemical Education - IUPAC, n. 26.).

VARGAS, M. (1994). Para uma filosofia da tecnologia. São Paulo: Alfa Omega.

WAKS, L. J. (1990). Educación en ciencia, tecnología y sociedad: origenes, desarrollos internacionales y desafíos actuales. In: MEDINA, M., SANMARTíN, J. (Eds.). Ciencia, tecnología y sociedad: estudios interdisciplinares en la univeridade, en la educacíon y en la gestión política y social. Barcelona, Anthropos, Leioa: Universidad del País Vasco.

WAKS, L. J., BARCHI, B. A. (1992). STS in U.S. school science: perceptions of selected leaders and their implications for STS education. Science Education, v. 76, n. 1, p.79-90.

YAGER, R. (1990). Science, technology, society: a major trend in science education. In: UNESCO. New trends in integrated science teaching. Bélgica: UNESCO, p.44-48.

YAGER, R. E., TAMIR, P. (1993). STS approach: reasons, intentions, accomplishments, and outcomes. Science Education, v. 77, n. 6, p.637-658.

ZOLLER, U. (1982). Decision-making in future science and technology curricula. European Journal of Science Education, v. 4, n. 1, p.11-17.

ZOLLER, U., WATSON, F. G. (1974). Technology education for nonscience students in the secondary school. Science Education, v. 58, n. 1, p.105-116.

ZOLLER, U., DONN. S., WILD, R., BECKETT, P. (1991a). Students' versus their teachers' beliefs and positions on science/technology/society - oriented issues. International Journal of Science Education, v. 13, n. 1, p.25-36.

. (1991b). Teachers' beliefs and views on selected science/technology/society - topics: a probe into STS literacy versus indoctrination. Science Education, v. 75, n. 5, p.541-561.

\section{Correspondência:}

Prof. Wildson L. P. dos Santos, Universidade de Brasília, Instituto de Química; Campus Universitário Darcy Ribeiro - Asa norte - Brasília, DF - cep 70910-900. Correio eletrônico: wildson@unb.br

Prof. Eduardo Fleury Mortimer, Universidade Federal de Minas Gerais, Faculdade de Educação, Cecimig; Av. Antônio Carlos, 6627 - Belo Horizonte, MG - cep 31270-901. Correio eletrônico: mortimer@dedalus.lcc.ufmg.br 